\title{
Evaluation of Enamel Loss with Polụcrusstalline Ceramic Bracket using two different Debonding Techniques
}

\author{
Dr P Narayana Prasad,' Dr Tarun Sharma, ${ }^{2}$ Dr Gaurav Chaudhary, ${ }^{3}$ Dr Arjun Vedvyas,, Dr Sandeep Gupta \\ Professor, 23.4Associate Professor, sPost Graduate Student \\ Department of Orthodontics, Seema Dental College, Rishikesh, India \\ Correspondence: Dr Arjun Vedvyas; Email: dr.arjunvedvyas@gmail.com
}

\section{ABSTRACT}

Introduction: Debonding of ceramic brackets requires a technique that will restore the enamel surface possibly to its pretreatment condition.

Objective: To evaluate a comparative degree of enamel loss with various debonding techniques.

Materials \& Method: 30 extracted premolars were divided into three equal groups of 10 each on the basis of bonding material used. Six subgroups comprising of 5 teeth each were formed on the basis of debonding techniques. Debonded enamel surfaces were photographed under Scanning Electron Microscope to evaluate the enamel loss percentage and compared using oneway ANOVA.

Result: Decreasing order of enamel surface loss was $63.1 \%, 56.3 \%, 45.4 \%, 43 \%, 31 \%$ and $30.5 \%$ for light cure-mechanical, light cure-electrothermal, self cure-mechanical, self cure-electrothermal, single composite-mechanical and single compositeelectrothermal combination respectively.

Conclusion: Mechanical debonding leads to greater enamel loss than electrothermal debonding. Bonding with Light cure composite always leads to greater enamel loss irrespective of type of debonding technique. Bonding with single composite always leads to lesser enamel loss irrespective of debonding technique.

Key words: ceramic bracket, debonding, enamel

\section{INTRODUCTION}

Fixed orthodontic treatment is the most common and extensively used treatment option for various types of malocclusions. Over the years, there has been considerable advancement in the materials used in orthodontic treatment. ${ }^{1-2}$ Ceramic brackets are being used extensively in modern orthodontic practice because of their higher esthetics and greater bond strengths over conventional metallic brackets..$^{3-6}$ During the course of treatment there is a need of bonding the brackets with bonding agent that will have adequate bond strength between the tooth and bracket so that it will resist force applied to the teeth. ${ }^{7-8}$

The term debonding refers to removal of orthodontic attachments and residual adhesive from the enamel surfaces that restore as closely as possible to its pre-treatment condition without inducing iatrogenic enamel damage. ${ }^{3}$ During debonding it should be ensured that natural structure of the tooth remains intact or minimal tooth enamel loss shall occur.

This study facilitates in recognizing a bonding agent that will have sufficient bond strength throughout the treatment period, and when it is debonded, it shall cause minimal loss of tooth enamel. The study identifies a technique of debonding that will cause minimal tooth enamel loss. Debonding is required at the termination of active treatment. Great consideration should be given to debonding procedures and their effect on the enamel surface underlying the bonded attachments. Primary consideration lies in the returning the enamel surface to as near as its original state as possible following the removal of bonded orthodontic attachments.

The purpose of the study was to microscopically examine the enamel surface structure subjected to different debonding techniques using Scanning Electron Microscope (SEM) to identify a technique that restores the enamel surface as closely 
as possible to natural enamel surface. Thus the objective of this study was to identify a debonding technique that will cause minimal enamel loss and to evaluate a comparative degree of enamel loss with various debonding techniques.

\section{MATERIALS AND METHOD}

Thirty premolars (extracted for therapeutic purpose) were collected. Tooth that have any type of enamel loss, carious lesion, hypoplastic or fluorosed enamel, fractured or treated were not included in the study. Teeth were stored in storage medium containing $10 \%$ formalin solution. Prophylaxis of enamel surface was performed by using rubber cup (Microdont Soccoro, Brazil) and fine pumice powder (SS white) and rinsed with water at low speed for 10 second and dried with oil-free compressed air. The center of clinical crown was then etched with $37 \%$ ortho-phosphoric acid (Meta Etchant) for $30 \mathrm{sec}$, rinsed for $30 \mathrm{sec}$ and dried for $10 \mathrm{sec}$ with compressor air. ${ }^{5-6}$

After etching, a fine and uniform layer of primer adhesive (Transbond XT, 3M Unitech) (except for single composite group) was applied with single stroke brushing and spreaded with short burst of moisture-free air blow. ${ }^{8-9}$ After application of the primer, each group was loaded with deferent bonding material which was placed at the long axis centre of clinical crown (this is done for the best fit of the bracket base and to ensure its relevance to clinical situation) and applying the force of 300 grams allowing the excess material to flow off producing a resin layer of uniform thickness. ${ }^{10-13}$

Bracket/enamel interface was cured with LED light curing unit at a distance of $5 \mathrm{~mm}$ for 10 second at the mesial surface and $10 \mathrm{sec}$ at the distal surface. ${ }^{13}$ Each group was divided into two subgroups containing 10 samples and one subgroup of each group was debonded mechanically using debonding plier (Ormco, USA) and other subgroup was debonded electrothermally; the temperature should not be exceeded more than $160^{\circ} \mathrm{C} .{ }^{12}$ Each group was debonded after 24 hours of bonding. ${ }^{1-3}$

Bracket removal was done using the debonding plier in such a way that the plier was placed at the base of the bracket, and a slight amount of squeezing pressure applied to the handles of plier until debonding occurred. The direction of applied force was occluso-gingival. In electrothermal debonding (ETD) of brackets from the tooth surface; electrical device (Figure 1) was used to generate heat and that heat is transferred to the bracket base by a blade of electrothermal device that is placed at the bracket slot at less than $160^{\circ} \mathrm{C} .^{12}$

When the heat is applied to the bracket, heat is transferred and deformed the adhesive bracket interface then the bracket could be easily lifted without excessive force to underlying enamel surface. ${ }^{12}$ Before removing excess adhesive and polishing the enamel surfaces, each specimen was assessed for enamel loss under the magnification with scanning electron microscope (SEM) for surface enamel loss. ${ }^{10}$ Before keeping the samples inside the sputter coater (Emitech sc-7620 Quaram technology) (Figure 2) for gold palladium coating sample was dried in incubator and dessicator (Figure 3). Enamel surface following debonding were examined under SEM (Figure 4); which were viewed in computer monitor (Figure 5).

Evaluation was done by measuring the surface area of enamel under the bracket then lost enamel surface was measured for each sample. Then mean was calculated for one subgroup then percentage loss of enamel was calculated and this percentage loss was compared and analyzed by ANOVA for intra-group and paired t-test for inter-group variation among all six subgroups.

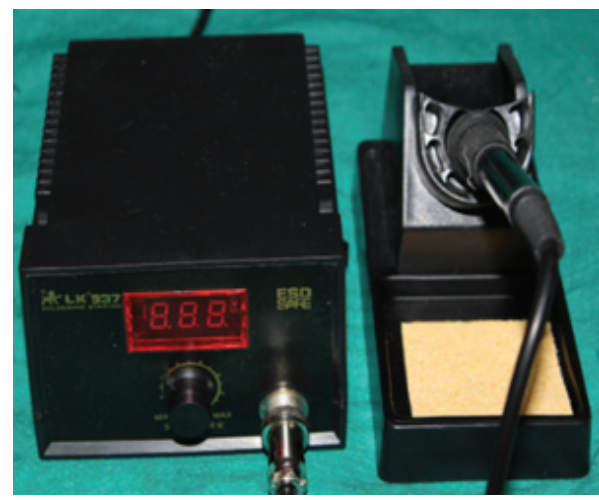

Figure 1: Electrothermal device with temperature display

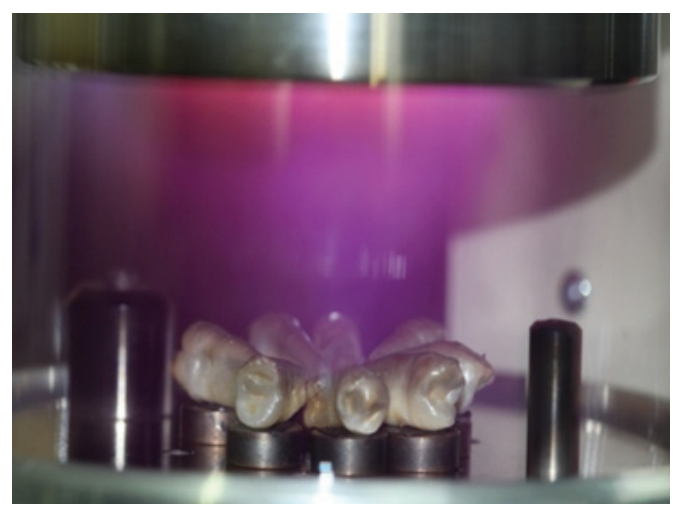

Figure 2: Sputter coater

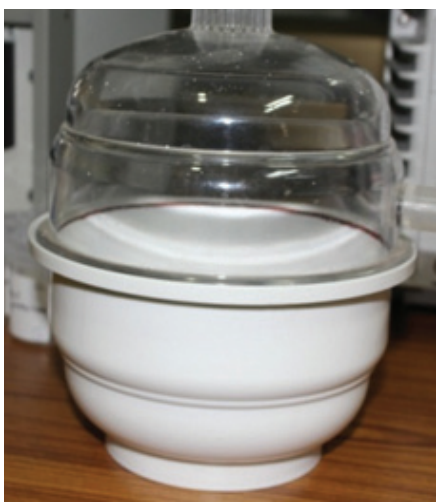

Figure 3: Dessicator 


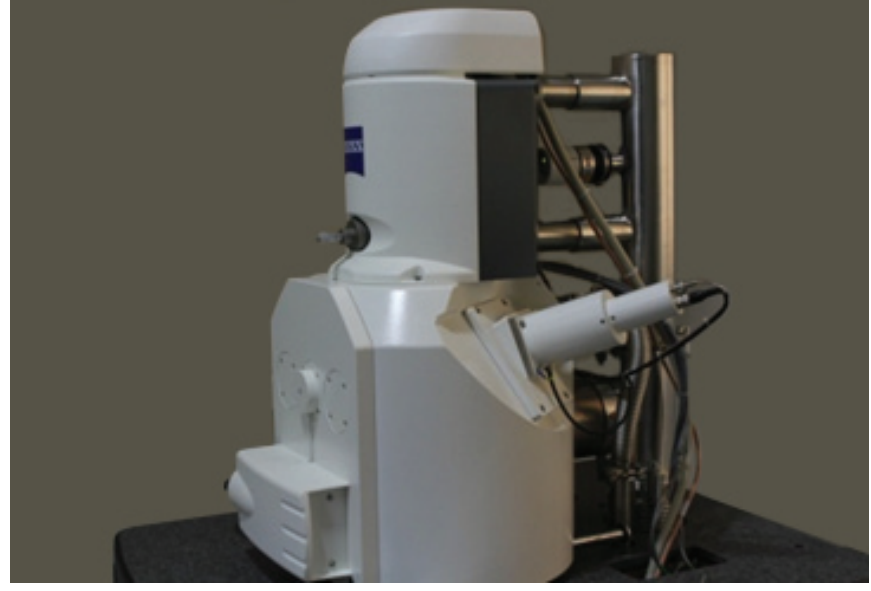

Figure 4: Scanning Electron Microscope

\section{RESULT}

For mechanical debonding using light cure composite bond material, surface loss was $63.1 \%$ (Figure 6a, 6b); for electrothermal debonding using light cure composite bond material, enamel surface loss was $56.3 \%$ (Figure $7 a$ 7b). For mechanical debonding using self cure composite

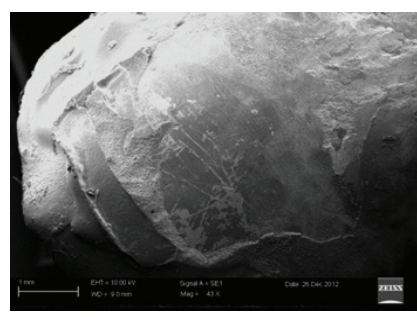

Figure 6a-Enamel surface after mechanical debonding using light Cure composite

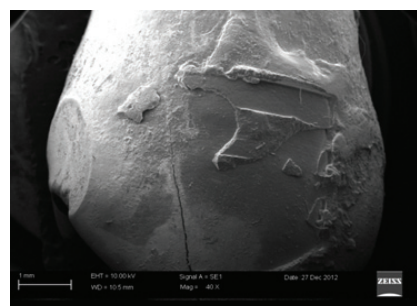

Figure 8a: Enamel surface after mechanical debonding using self cure composite bond material

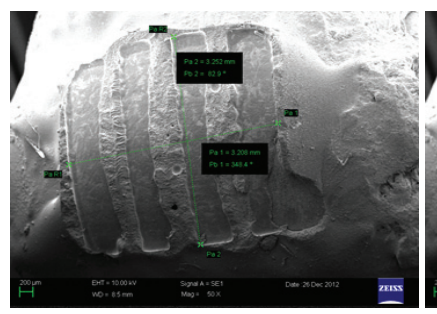

Figure 10a: Enamel surface after mechanical debonding using single composite bond material

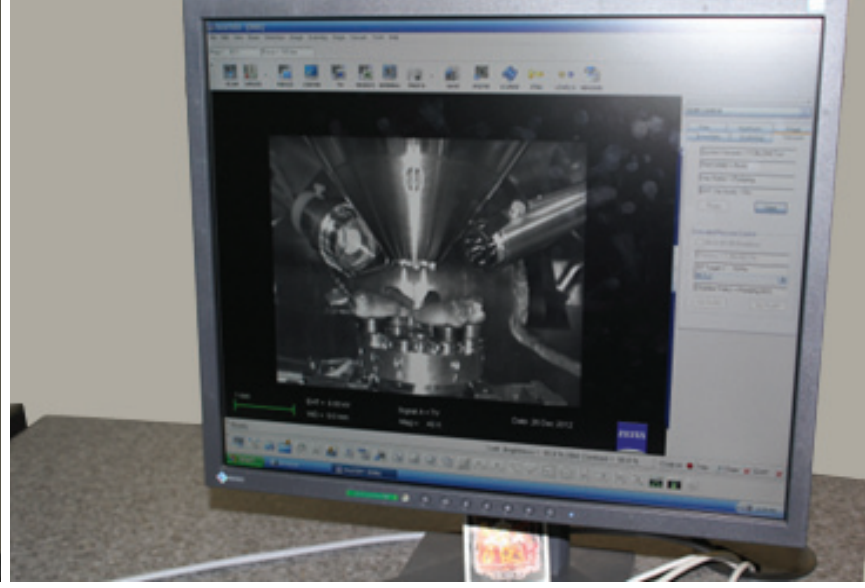

Figure 5: Teeth sample in computer monitor

bond material, surface loss was $45.4 \%$ (Figure $8 a, 8 b$ ), for electrothermal debonding using self cure composite bond material, surface loss was 43.4\% (Figure 9a, 9b). For mechanical debonding using single composite bond material, surface loss was $31.0 \%$ (Figure 10a, 10b), for electrothermal debonding using single composite bond material, surface loss was $30.5 \%$ (Figure 11a, 11b).

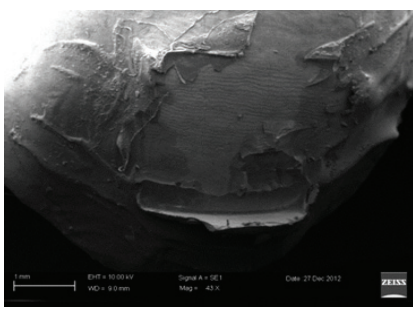

Figure 7a: Enamel surface after electrothermal debonding using light cure composite

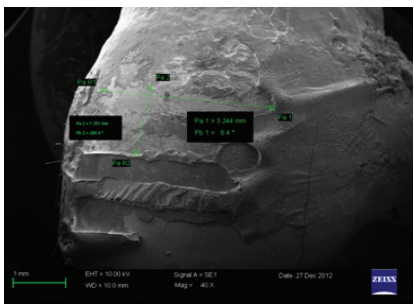

Figure 9a: Enamel surface after electrothermal debonding using self cure composite bond material

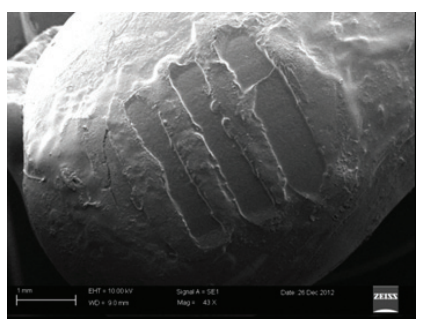

Figure 11a: Enamel surface electrothermal debonding using single composite

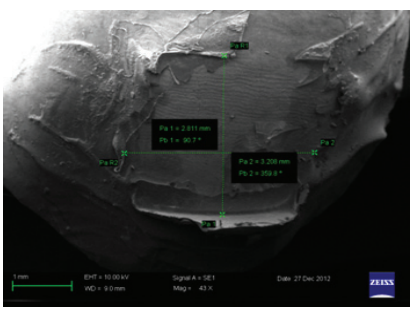

Figure 7b: Measurement of enamel loss

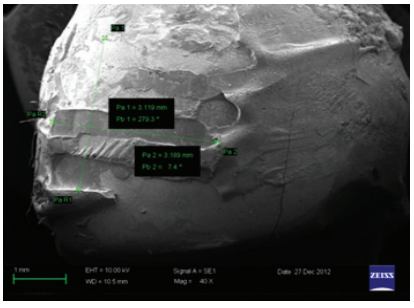

Figure 9b: Measurement of enamel loss

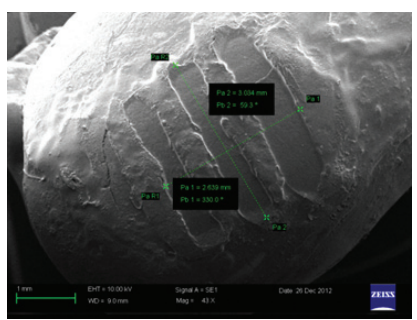

Figure 11b: Measurement of enamel loss 


\section{DISCUSSION}

Debonding of bracket always leads to enamel loss and it depends upon the type of bonding material and debonding technique used. Removal of ceramic brackets using mechanical debonding technique always leads to greater enamel loss than electro-thermal debonding. Following the etching; bonding is achieved mainly by the formation of resin tags, hence when we debond mechanically it leads to breakage of the resin tags along with the adjacent enamel structure, leading to more enamel loss compared to electrothermal debonding i.e. in which the heat delivered by the instrument weakens the resin tags and allow easy removal and less enamel damage.

Bonding with light cure composite causes greater enamel loss irrespective of type of debonding technique used.
Bonding with single composite always lead to lesser enamel loss irrespective of debonding technique used. Although the bond strength of single composite have sufficient bond strength for orthodontics but it is very less as compared to the light cure however, it is easy to remove and lesser enamel damage is reported with single composites.

\section{CONCLUSION}

Maximum enamel loss occur during mechanical debonding using light cure composite bonding material and minimum enamel loss occur during electro thermal debonding using single composite bonding material.

\section{REFERENCES}

1. Chaconas SJ, Angelo AS, Gary LN. Bond strength of ceramic brackets with various bonding systems. Angle Orthod 1990; 61:35-42.

2. Eliades T, Aanthony D, Lekka M. Failure mode of analysis of ceramic brackets bonded to enamel. Am J Ortho Dentofac Orthoped 1993 Jul; p21-26

3. Meguel JAM, Almeida MA, Chevitarese O, Hill C. Clinical comparison between glass-ionomer cements and composite for direct bonding of orthodontic brackets. Am J Ortho Dentofac Orthoped 1995; p484-487.

4. Miller R.J, Manci L, Arbuckle, Baldwin J, Philips RW. A three clinical trial using a glass inomer cement for the bonding of orthodontic brackets. Angle Orthod 1996; 66:4:309-312.

5. Sinha PK, Nanda RS. The effect of different bonding and debonding technique in debonding ceramic orthodontic brackets. Am J Ortho Dentofac Orthoped 1997 Aug; p132-135.

6. Kearns PO, Sandham JA, Jones WB. Electrothermal deboning of ceramic brackets; Br J Orthod 1997; 24:237-242.

7. Cacciafesta $V$, Subenberger U, Brinkenmann J, Miethke. Shear bond strength ceramic brackets bonded with different light cured glassinomer cements. Euro J Orthod 1998; p177-187.

8. Arici S, Minors C. The force level required to mechanically debond ceramic brackets. Euro J Orthod 2000; 22:327-334.

9. Ireland AJ, Hosein I, Sherriff M. Enamel loss at bond up and clean following the use of a conventional light cured composites and resin modified glass-inomer cement. Euro J Orthod 2005; 27:413-419.

10. Uma HL, Chandralekha B. A Scanning electron microscopic evaluation of the enamel surface subsequent to various debonding procedures. Achieve of Oral Science \& Research 2012; p7-22.

11. Pignatta LMB and Durate S. Evaluation of enamel surface after bracket debonding and polishing. Dental Press Journal Orthod.2012 77-84.

12. Sheridan JJ, Brawley G and Hasting J. Electrothermal debracketing Part II: An vivo study. Am J Orthod 1986; $142-145$.

13. Bishara SE, Ajlouni R, Warren J. Effect of modifying the adhesive composition on the bond strength of orthodontic brackets. Angle Orthod 2002; 70:352-6. 